\title{
Mathematical modeling of chilling stress induced changes in Arabidopsis thaliana root meristem
}

\author{
M.S. Savina ${ }^{1,2 *}$, J.H. Hong ${ }^{3}$, Jian Xu $\mathrm{Xu}^{3}$, V.V. Mironova ${ }^{1,2}$ \\ ${ }^{1}$ Institute of Cytology and Genetics SB RAS, Novosibirsk, Russia \\ ${ }^{2}$ Novosibirsk State University, Novosibirsk, Russia \\ ${ }^{3}$ Department of Biological Sciences and Centre for BioImaging Sciences, National University \\ of Singapore, Singapore, Singapore \\ *e-mail: savina@bionet.nsc.ru
}

Key words: mathematical modeling, developmental biology, chilling stress, hormone regulation

Motivation and Aim: Plant hormone auxin guides many physiological processes, including of stem cell niche maintenance in a changing environments. In Arabidopsis, chilling stress $\left(24 \mathrm{~h}\right.$ under $\left.4{ }^{\circ} \mathrm{C}\right)$ leads to DNA damage predominantly in root stem cells and their early descendants. However, only newly generated/differentiating columella stem cell daughters (CSCDs) preferentially die in a programmed manner. Inhibition of the DNA damage response in these CSCDs prevents their death but makes the stem cell niche more vulnerable to chilling stress. We studied the protective effect of CSCD death on the stem cell niche under chilling stress in a mathematical model.

Methods and Algorithms: As a readout, we analyzed the expression levels of DR5::GFP, PIN::PIN-GFP, and PIN::GUS at 4 and $22{ }^{\circ} \mathrm{C}$. Chilling stress significantly alters expression of auxin transporters and lead to decrease in auxin response in the QC. For this study, we carry out computer modeling of the auxin distribution taking into account the positive and negative feedbacks between the auxin and its PIN transporter in the Arabidopsis thaliana root tip [1].

Results: Consistent with the experimental findings, in silico analysis showed that differential changes in the expression of PINs led to a new steady-state equilibrium of auxin distribution in chilling-stressed root, despite an overall decline in auxin levels. In this new steady state, however, the division of CSCs caused a loss of auxin maximum in the QC, which could be restored only if the death of newly generated CSCDs occurred. By contrast, CSC division at normal temperature had no effect on the maintenance of auxin maximum in the QC. We thus concluded that CSCD death was the strategy used by the root to sustain the auxin maximum.

Conclusion: In agreement with our model prediction, that CSCD death increases the auxin concentration in the QC, roots with chilling stress-induced CSCD death displayed a higher DR5::GFP and WOX5::GFP expression in the QC than those without. Together, our findings demonstrate the importance of auxin level in the protection of root stem cell integrity and indicate that chilling stress-induced death of CSCDs results in an increase of auxin levels in the root stem cell niche, which helps prepare the root to withstand the accompanying environmental stresses and to recover faster when returned to optimal temperatures [2].

Acknowledgements: The work was supported by MK-1297.2017.4.

References

1. Mironova V.V. et al. (2012) Combined in silico/in vivo analysis of mechanisms providing for root apical meristem self-organization and maintenance. Annals of Botany. 110(2):349-360.

2. Hong J.H., Savina M. et al. (2017) A sacrifice-for-survival mechanism protects root stem cell niche from chilling stress. Cell. 170(1):102-113. 